Research Article

\title{
Design and Application of Science and Technology Project Management Information System for Educational Institutes
}

\author{
Jinhua Liu $(\mathbb{D}$, Caiping Wang, and Xianchun Xiao \\ Jiangsu Vocational College of Agriculture and Forestry, Jurong, Zhenjiang 212400, China \\ Correspondence should be addressed to Jinhua Liu; liujinhua@jsafc.edu.cn
}

Received 20 August 2021; Revised 13 September 2021; Accepted 14 September 2021; Published 25 September 2021

Academic Editor: Fazlullah Khan

Copyright (c) 2021 Jinhua Liu et al. This is an open access article distributed under the Creative Commons Attribution License, which permits unrestricted use, distribution, and reproduction in any medium, provided the original work is properly cited.

\begin{abstract}
This paper analyzes the user needs of the scientific research project management system, expounds the feasibility of information system development, and analyzes the business needs combined with the reality of scientific research project management. In the system level design, combined with the demand analysis of the information system function, the system module is developed in a hierarchical way. The whole system is divided into four layers: (i) core business layer; (ii) data access interface layer; (iii) neighborhood object layer; (iv) data platform. The specific functions include three various modules, i.e., scientific research project information management module, research achievement management module, and scientific research information portal module. In the system software part, the attribute encryption method is used to effectively encrypt the information of scientific and technological projects and to protect the safe sharing of scientific and technological information in colleges and universities. Finally, we look at the limitations of conducting high-quality design-science research in the context of the larger information system community. The experimental results show that the system can perform the corresponding functions effectively, and the data reading integrity is high, which can meet the requirements of the university science and technology project information management.
\end{abstract}

\section{Introduction}

Universities will accelerate the formation of a new normal of scientific research supervision through establishing a longterm mechanism for scientific research inspection and further improving the management system and mechanism of scientific research funds. Moreover, educational institutes give full play to the main role of universities in their own scientific research management and supervision, improve the level of scientific research management, and promote the reform of the scientific and technological system in colleges and universities. These factors can help all educational institutes in promoting their healthy and sustainable development. How to manage all kinds of information of scientific research projects, how to evaluate the service quality of scientific research projects, and how to provide data basis for decision-making in colleges and universities are the focus of scientific research management in colleges and universities [1-3]. With the more and more in-depth and extensive application of information technology in management, the implementation of the management information system has gradually matured in the technology. Efficient scientific research management information system helps scientific research managers and teachers get rid of heavy tasks.

The quality of scientific research management in colleges and universities plays an important role in the development of country economy, education systems, and better human lives. It is still one of the most important components to reflect the reform and development of colleges and universities and the establishment of informatization. With the increasing number of scientific research tasks in colleges and universities year by year, the increase of funds, and the emergence of diversified achievements, new challenges and requirements are put forward for scientific research management. It is urgent for scientific research management departments in colleges and universities to make scientific research project management more systematic, 
standardized, and efficient. The construction and use of scientific research management information system have practical significance that cannot be ignored to improve the scientific research management level of colleges and universities and better serve the scientific research improvement of colleges and universities [4].

How to further strengthen the management of scientific research information, improve the quality of scientific research information management, enhance work efficiency, and reduce the cost of scientific research management is an important topic facing the scientific research management of colleges and universities in various countries including China [5-7]. Through the demand analysis of scientific research management in colleges and universities, based on the in-depth analysis of the current situation of scientific research management in colleges and universities, this paper expounds the development background and development environment of the system and analyzes and studies the framework of the system. Moreover, the article analyzes the integrity of the system design and studies the data consistency and information security. The main contributions of our work are as follows:

(i) We analyze the user needs of the scientific research project management system and expound the feasibility of information system development

(ii) We analyze the business needs combined with the reality of scientific research project management. The system module is developed in a hierarchical way.

The structure of this paper is organized as follows. In Section 2, we introduce the architecture of science and technology project management information system in colleges and universities. In Section 3, we present the system hardware design. Similarly, Section 4 describes the system software verification. Finally, we conclude the paper in Section 5 along with few directions for further research and investigation.

\section{Science and Technology Project Management Information System Architecture}

According to the survey, the management system of colleges and universities has been basically realized. Most colleges and universities have launched educational administration management, financial management, and logistics management systems, including the scientific research information management system, which has realized the integration of various systems and has become one of the important components of digital campus construction [8-10]. Scientific research information management in colleges and universities refers to the collection, sorting, organization, storage, processing, use, and maintenance of scientific research information in colleges and universities. Before construction, in the demand analysis, according to the needs and possibilities, based on the principle of "revitalizing the university through scientific research" and using advanced computer information technology, we should realize the information sharing of scientific research information management, application, audit, office, and so on. The purpose is to integrate resources, make more rational use of and share scientific research information resources, and achieve the management effect of scientific research management, scientific research learning, and automation.

The scientific research information management system of colleges and universities can be divided into scientific research office, organization, project management, fund management, scientific research achievements, academic exchange, assessment and reward, statistical report submission, system management, and other parts [11]. Each module has a subordinate multilevel management subsystem. The subsystems are related to each other and relatively independent, which can facilitate colleges and universities to select and implement important projects. A taxonomy of various modules of the university science and technology project management information system is shown in Figure 1.

Practicability is the most important index in the development of university science and technology information management system. Meeting the needs of scientific research management is the primary task in the system development and design [12-14]. In the design concept, the primary consideration is the convenience of users. A set of standards should be formulated to unify coding rules and interface style and Chinese format, as well as good maintainability, simplicity and ease of use, humanized design, functional integrity, diversity, and selection, so as to provide users with access services. The specific design and implementation of the university scientific research management system are to analyze and integrate all information in scientific research management on the basis of comprehensive use of information technology and then carry out the optimal allocation of existing resources, so as to create an automatic office system integrating information audit, query, and so on for scientific research management workers and university teachers; then, it creates good conditions for the system to ensure its maintainability and integrity in future application. The basic sharing relationship between science and technology project management information system and other systems is shown in Figure 2.

The system chooses the most widely used Windows operating system as the platform for research and development and considers using Microsoft's Visual Basic.NET to ensure the compatibility of application development. The database chooses SQL Server 2000 for development. Compared with miniaturized database systems such as My SQL, SQL Server database management system is relatively more friendly. It is a powerful MIS system development tool that is easy to use, simple to develop, and flexible in interface. Visual Basic.NET provides a visual programming environment, a convenient and fast way to develop Windows applications for everyone [15]. Compared with ordinary database systems, using Visual Basic.NET as a database development platform has the characteristics of simplicity, flexibility, scalability, etc., which are very suitable for beginners. At present, the development of common 


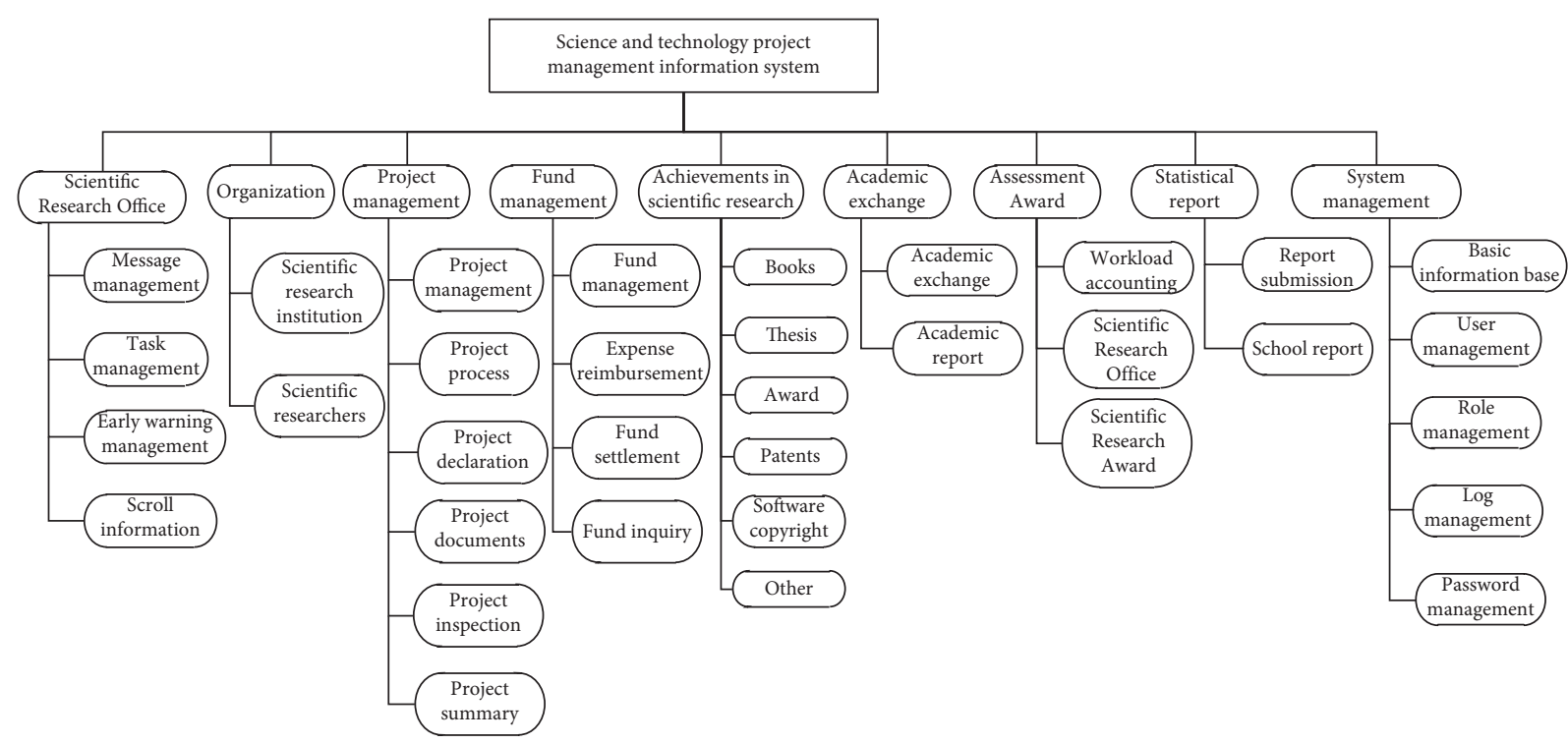

Figure 1: Modules of the project management information system.

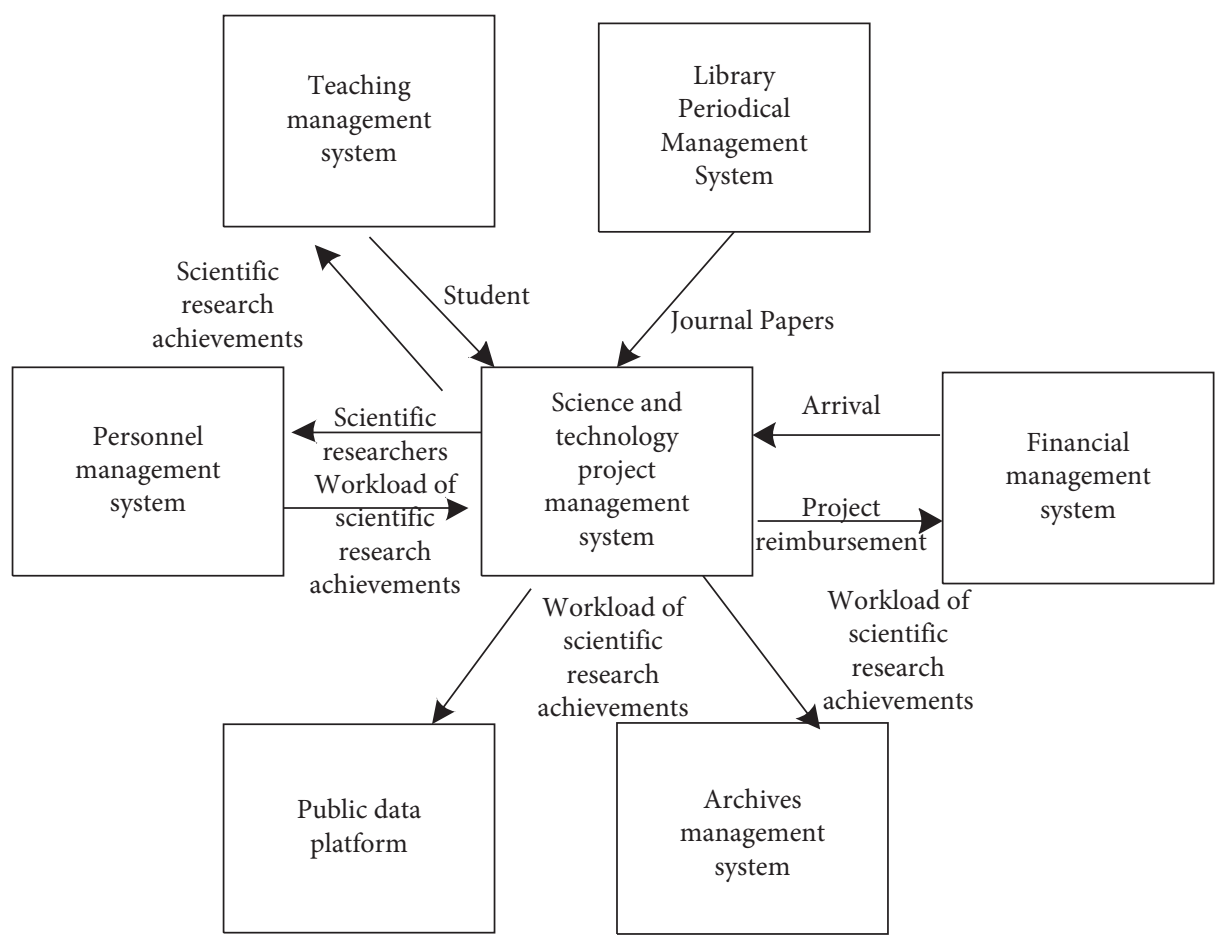

FIgURE 2: Relationship between science and technology project management information system and other systems.

management systems is implemented in large areas by using object-oriented and database-based technology. This technology method is currently very mature and is being widely implemented in various industries.

The university scientific research management information system should be a network-based management information system, which serves the personnel engaged in scientific research or scientific research management of the whole university and can customize other corresponding business processes according to business needs. Various users work together through the network. Its overall design framework is shown in Figure 3.

\section{System Hardware Design}

The system hardware design comprises three modules. Discussion around each module is provided in subsequent sections. 


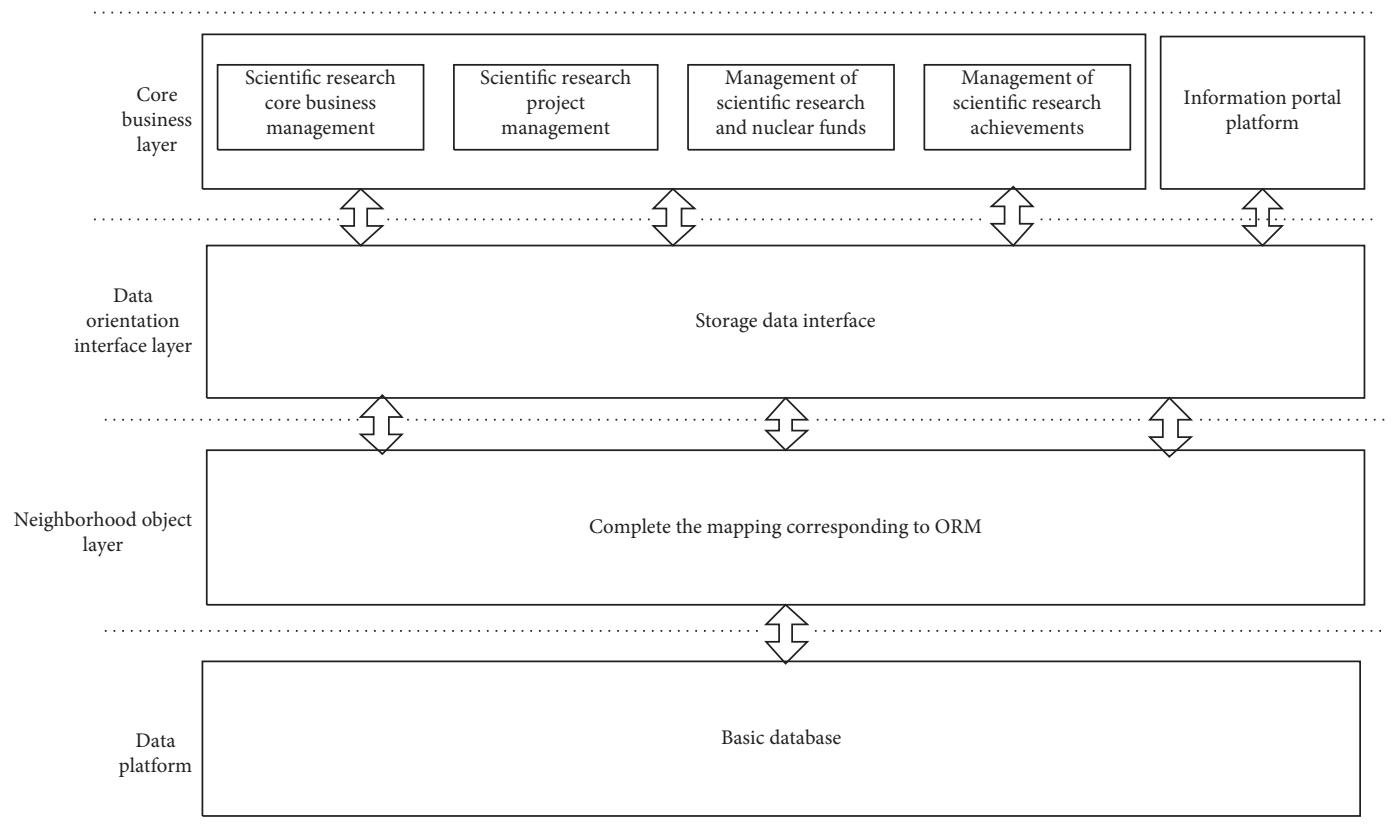

Figure 3: Overall system design architecture.

3.1. Scientific Research Project Information Management Module. Taking the modification of scientific research project information by administrators and superusers as an example, the following sequence diagram gives a detailed design description of this operation. The sequence diagram of scientific research project information modification is shown in Figure 4.

Combined with the sequence diagram, when the administrator needs to modify the scientific research project information, first enter the correct username and password to enter the system and select the scientific research project information modification function, enter the modification interface, and select the information items that can be modified, and the system control BasicinformationAction.java accepts the user's modification request, verifies the validity of the request, and highlights the fields that the user has modified. After confirming the modified information, the user clicks the "Save" button on the interface, and the system calls the save() method in the business logic class BasicinformationManager.java, interacts the modified data with the entity class Basicinformation.java, and replaces the original related information in the database. The information is modified and updated, and finally, the encapsulated modification result is returned, which is processed by the system control class BasicinformationAction.java, and finally, the modification success or failure information is displayed on the foreground user interface.

\subsection{Scientific Research Achievement Management Module.} The scientific research achievement management module is mainly aimed at scientific researchers and scientific research managers at all levels. The types of scientific research achievements corresponding to the system mainly include scientific research papers, scientific research works, scientific research awards, scientific research patents, software copyrights, and other forms of scientific research achievements. The functions of the scientific research achievement module of the system are shown in Figure 5.

3.2.1. Input of Scientific Research Achievements. Scientific research achievements are mainly entered by scientific researchers according to the type of scientific research achievements given by the system.

3.2.2. Review of Scientific Research Achievements. The review of scientific research achievements is mainly completed by scientific research managers. Scientific research managers review the scientific research achievements entered by scientific researchers. Only the approved achievements are included in the achievement statistics of individuals or scientific research departments.

\subsubsection{Query and Statistics of Scientific Research} Achievements. Scientific researchers and scientific research managers can query scientific research achievements. The difference is that scientific researchers can only find individual scientific research achievements, while scientific research managers of colleges and departments can view all scientific research achievements in their own department, and scientific research managers of schools can view all scientific research achievements of the whole school. Scientific research managers can make statistics on the scientific research achievements of their departments. The statistical contents include displaying the number of scientific research achievements of their departments according to the year and achievement category and making statistics on the category of scientific research achievements according to the year. 


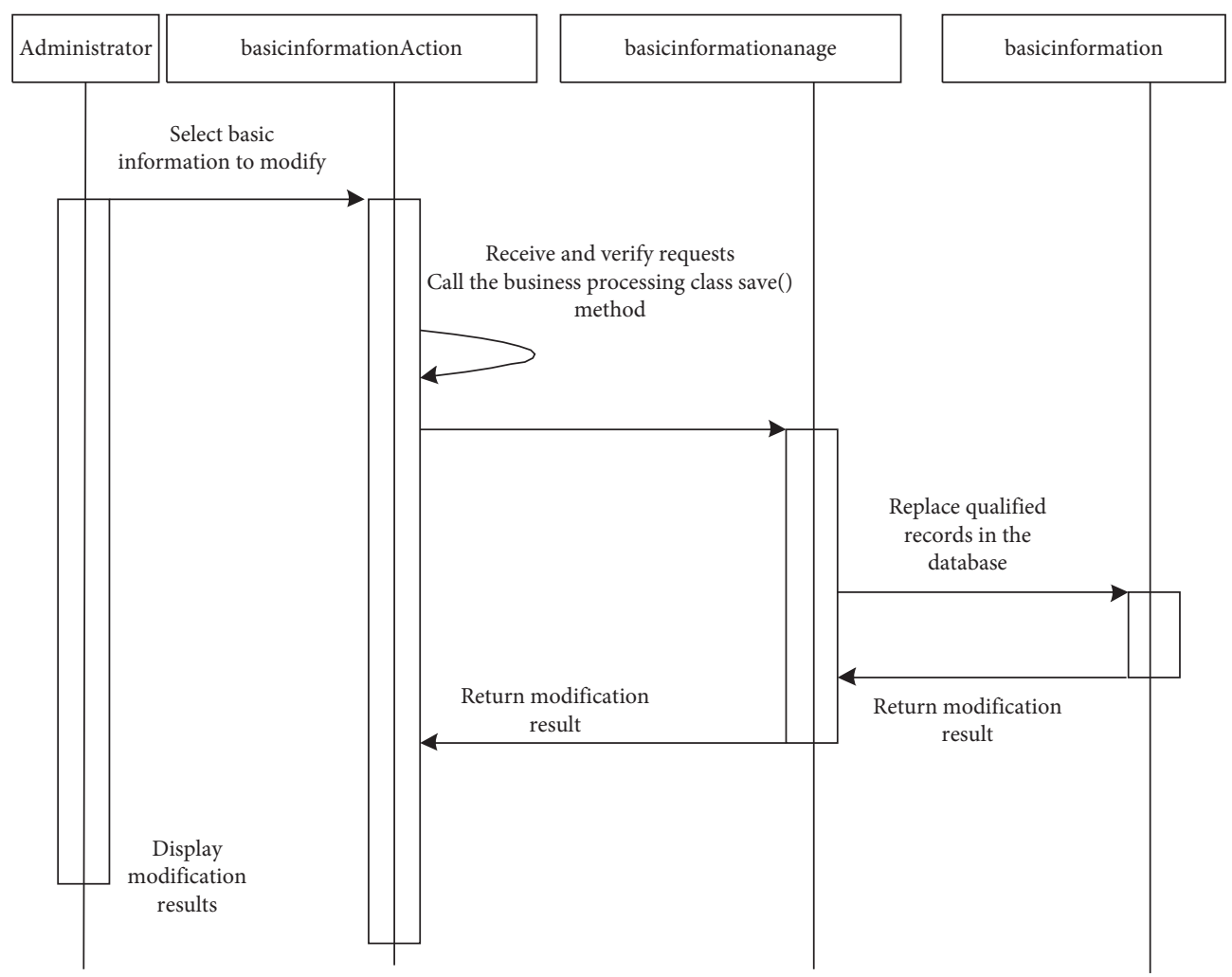

FIGURE 4: Sequence diagram of scientific research project information modification.

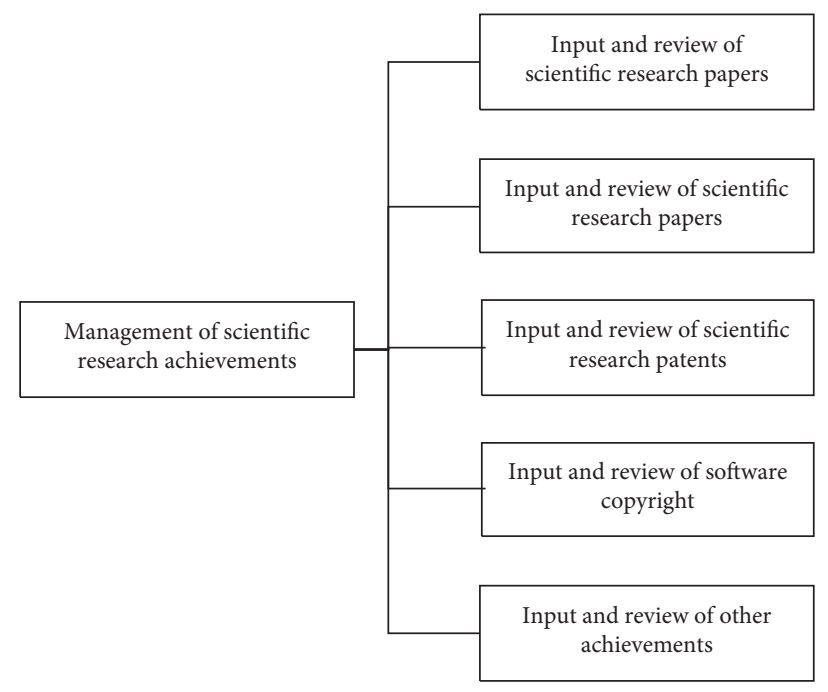

FIGURE 5: Scientific research achievement management module.

3.3. Scientific Research Information Portal Module. Scientific research information portal is a platform for the release and exchange of scientific research information in colleges and universities. This part is closely related to scientific research activities. It can timely and dynamically release scientific research news and scientific research notices, display scientific research trends, clarify work processes, and provide a login entry for business process processing. Scientific research management personnel can dynamically update and maintain the columns and contents of scientific research information portal. Ordinary tourist users can browse and retrieve relevant information. The retrieved content is consistent with the data of the whole university scientific research management information system. The specific block diagram of the scientific research information portal design is shown in Figure 6.

According to the demand analysis, the scientific research information portal module is divided into three parts: section display, column display, and login entry of core business. The management of the scientific research 


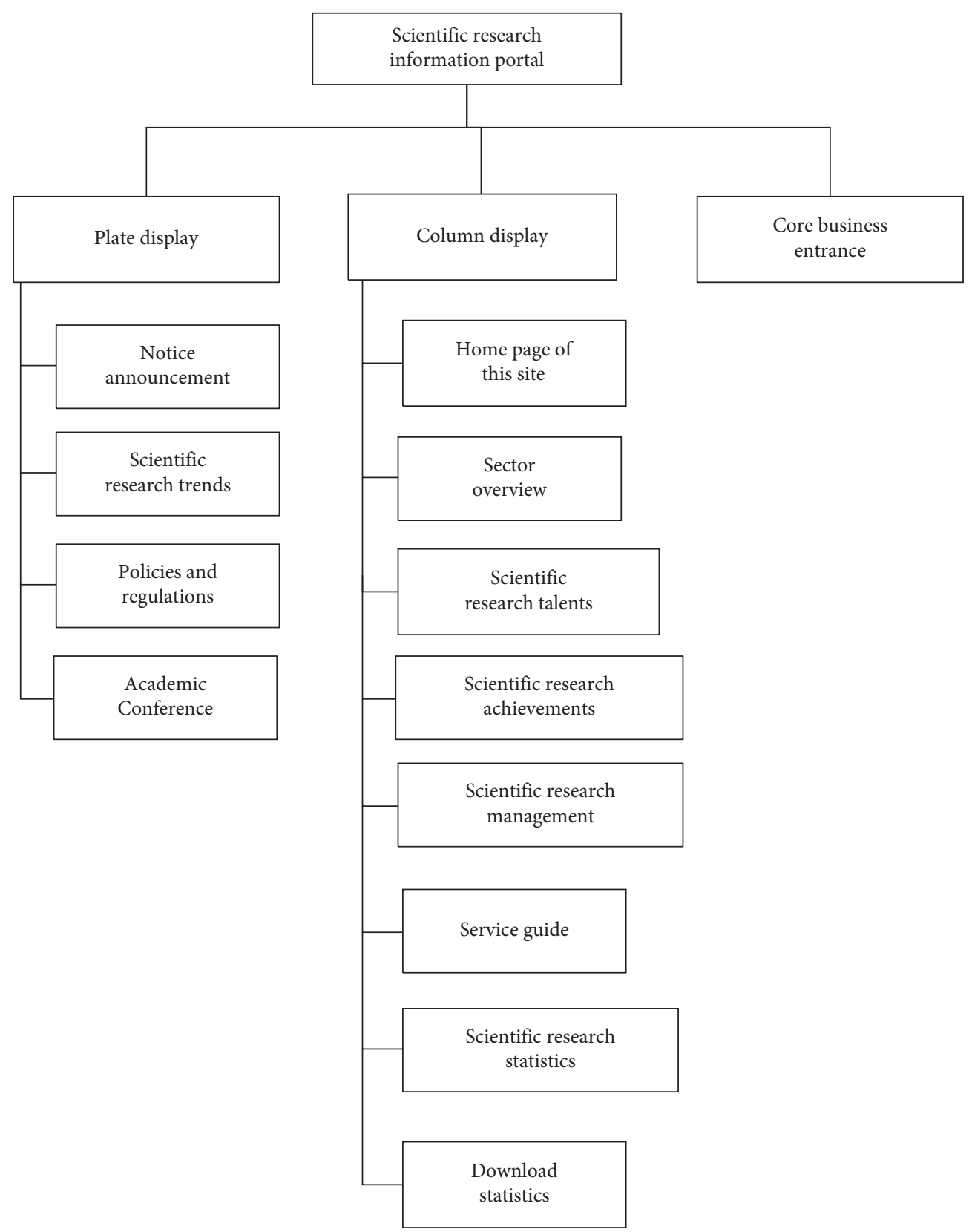

FIGURE 6: Functional design block diagram of the scientific research information portal.

information portal is managed by school-level scientific research managers, mainly including notification news release, column management, and other portal information management.

3.3.1. Section Display. The section display is the main display information of the scientific research portal, mainly including notices and announcements related to scientific research of the whole university, scientific research trends, policies and regulations, and academic conferences. By viewing the information of these sections, users can accurately grasp the overall situation of current scientific research and what academic conferences and activities there are.
3.3.2. Column Display. Column display is the main line running through the whole scientific research information portal, mainly including 8 columns: home page of the website, department overview, scientific research talents, scientific research achievements, scientific research management, service guide, scientific research statistics, and download area. Columns can be adjusted as needed and are generally managed and maintained by school-level scientific research managers.

3.3.3. Core Business Entrance. Users enter the corresponding business processing platform through the core business portal and conduct corresponding management and business processing on the business processing 
platform. The core business entrance mainly faces the scientific researchers and scientific research managers in colleges and universities.

\section{System Software Design}

Due to the strong confidentiality of scientific research projects, the attribute encryption algorithm is used to encrypt the information of scientific and technological projects in the software design part of the system.

Suppose $\left\{P_{1}, P_{2}, \ldots, P_{n}\right\}$ represents a set composed of $n$ participants, and the access structure $A$ is a set composed of nonempty subsets of $\left\{P_{1}, P_{2}, \ldots, P_{n}\right\}$, that is, $A \subseteq 2\left\{P_{1}, P_{2}, \ldots, P_{n}\right\} /\{\varnothing\}$. The set elements in $A$ are called authorized sets; otherwise, they are called unauthorized sets subject to certain conditions and limitations. If $A$ is monotonic, then for $\forall A=B$ and $C$, if $B \in A$ and $B \subseteq A$, then $C \in A$.

Attributes are information elements used to describe users. For example, the classroom has attributes such as job number, name, gender, department, and professional title. In this article, participants refer to attributes, so access structure $A$ contains a collection and list of authorization attributes. Unless otherwise specified, the access structure in this paper refers to monotonic access structure.

Suppose the tree $T$ represents the access structure tree, where each nonleaf node represents the threshold. For any internal node $x$, suppose it has $n u m_{x}$ child nodes and $k_{x}$ is the threshold, then $0<k_{x}<\operatorname{num}_{x}$. According to the definition, when $k_{x}=1$, the node represents "or" door; when $k_{x}=$ num $_{x}$, the node represents "and" gate. The leaf node $l$ in $T$ corresponds to an attribute, and its threshold value is $k_{l}=1$.

Let parent $(x)$ represent the parent node of node $x$, $\operatorname{attr}(x)$ represent the attribute corresponding to leaf node $x$, and index $(x)$ represent the number of child nodes of node $x$, which satisfies $1 \leq \operatorname{index}(x) \leq \operatorname{num}_{x}$. The access tree structure is shown in Figure 7.

If the secret sharing scheme on the collective set $P$ satisfies the following properties, the sharing scheme is called a linear secret sharing scheme:

(1) All shares can form a vector on domain $Z_{p}$

(2) There is a shared generation matrix $M$ of order $l \times n$ and a mapping $\rho$ from $\{1, \ldots, l\}$ to $P$ so that, for $\forall x=1, \ldots, l$, row $\rho(x)$ represents an entity $r$ in the entity set
Suppose $s \in Z_{p}$ represents the secret to be shared, and $r_{2}, \ldots, r_{n} \in Z_{p}$ is randomly selected to form $v=\left(s, r_{2}, \ldots, r_{n}\right)$. Then, $M_{v}$ is to obtain the vector composed of $l$ shares about $s$ by using the sharing scheme, where $\left(M_{v}\right)_{x}$ belongs to entity $\rho(x)$.

Assuming that the access scheme is the LSSS of $A$ on the access structure, $S$ is an authorized subset, and $I=\{x \mid \rho(x) \in S\} \subset\{1, \ldots, l\}$ is defined, then there is a constant set $\left\{w_{x} \in Z_{p}\right\}_{x \in I}$ such that $\sum_{x \in I} w_{x} M_{x}=$ $(1,0, \ldots, 0)$, so there is $\sum_{x \in I} w_{x} M_{x} v=\left(s, r_{2}, \ldots, r_{n}\right)$. $(1,0, \ldots, 0)^{T}=s$. These constant sets can be obtained in polynomial time according to matrix $M$.

The user can recover plaintext only if and only if the attribute matches the access structure. The detailed scheme is as follows:

Suppose that $G$ and $G_{T}$ represent two multiplicative cyclic groups with prime order $q, g$ is a generator of $G, e: G \times$ $G \longrightarrow G_{T}$ represents bilinear mapping, $\lambda$ represents system safety parameters, and $H:\{0,1\}^{*} \longrightarrow G$ is a hash function. Define the Lagrange coefficient: $\Delta i, S(x)=\prod_{j \in S, j \neq i}$ $(x-j / i-j)$, where $i \in Z_{q}$ and $S$ is a set. The scheme includes the following five algorithms:

(1) Setup: The system initialization process is as follows: input group $G$ and random selection of parameters $\alpha$ and $\beta \in Z_{q}$. Formula (1) is shown below:

$$
P K=G, g, h=g^{\beta}, f=g^{1 / \beta}, e(g, g)^{\alpha} .
$$

and the system key $M K=\left(\beta, g^{\alpha}\right)$. Among them, $f$ is used for the delegate algorithm and process.

(2) $\operatorname{Encrypt}(P K, M, T)$ : the algorithm uses access structure $T$ to encrypt data $M$.

From the root node to the leaf node, a polynomial $p_{x}$ is randomly selected for each node $x$ of $T$. The degree of the highest term of the polynomial $d_{x}$ is less than 1 than the threshold set by the node, that is, $d_{x}=k_{x}-1$.

Randomly select secret $s \in Z_{q}$ for root node $\mathfrak{R}$, satisfy the condition $p_{\mathfrak{R}}(0)=s$, and then, randomly construct the other $d_{\mathfrak{R}}$ points. For node $x$, the selected polynomial satisfies $p_{x}(0)=p_{\text {parent }(x)}$ (index $(x)$ ), and then, construct the remaining $d_{z}$ points, from top to bottom, and so on. Suppose $L$ represents the node set in the middle leaf of tree $T$, and calculate the ciphertext, as given by

$$
C T=\left(T, \widetilde{C}=M \cdot e(g, g)^{\alpha s}, C=h^{s}, \quad \forall l \in L: C_{l}=g^{p l(0)}, C_{l}^{d}=H\left(\operatorname{attr}(l)^{p l(0)}\right)\right)
$$




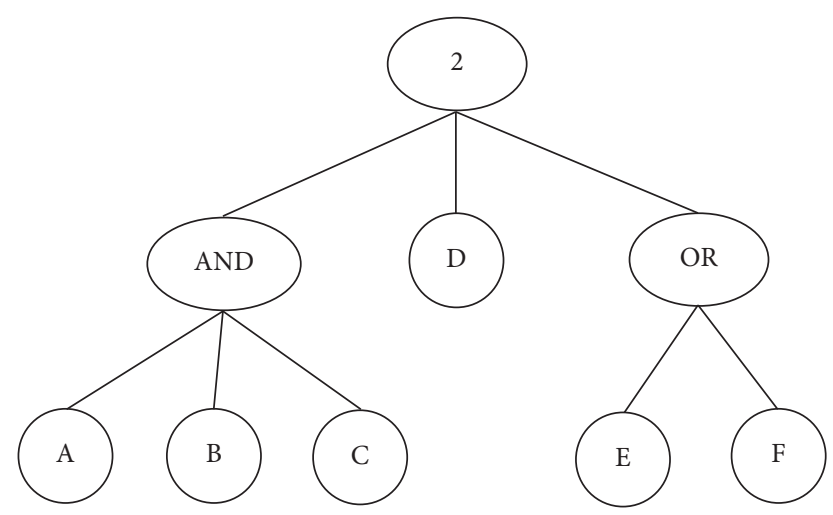

Figure 7: Access tree structure.

(3) KeyGen $(M K, S)$ : enter the attribute set $S$, and output $S$ and the corresponding user private key. Select $r \in Z_{q}$ randomly. For attribute $j \in S$, select $r_{j} \in Z_{q}$ to generate the user's private key, as illustrated using

$$
S K=\left(D=g^{(\alpha+r) / \beta}, \quad \forall j \in S: D_{j}=g^{r} \cdot H(j), D_{j}^{t}=g^{r j}\right) .
$$

(4) Delegate $(S K, \widetilde{S})$ : enter the private key $S K$ and attribute set $\widetilde{S}$ corresponding to attribute set $S$, where $\widetilde{S} \subseteq S$, and randomly select $\widetilde{r} \in Z_{q}$, and for $\forall k \in \widetilde{S}$, select $\widetilde{r}_{k} \in Z_{q}$ to calculate a new user private key that is computed using the formula given by

$$
\widetilde{S} \widetilde{K}=\left(\widetilde{D}=D \cdot f^{\bar{r}}, \quad \forall k \in \widetilde{S}: \widetilde{D}_{k}=D_{k} \cdot g^{\bar{r}} \cdot H(k)^{\bar{r}_{k}}, \widetilde{D}_{k}^{r}=D_{k}^{r} \cdot g^{\bar{r}_{k}}\right) .
$$

(5) Decrypt $(C T, S K)$ : firstly, define a recursive algorithm DecryptNode $(C T, S K, x)$, which inputs the ciphertext $C T=\left(T, \widetilde{C}, C, \forall l \in L: C_{l}, C_{l}^{t}\right)$ and the user's private key $S K$. The calculation process is as follows:

(a) If $x$ is a leaf node, let $i=\operatorname{attr}(x)$. If $i \in S$, then

$$
\operatorname{DecryptNode}(C T, S K, x)=\frac{e\left(D_{i}, C_{x}\right)}{e\left(D_{i}^{\prime}, C_{x}^{\prime}\right)}=e(g, g)^{r p_{x}(0)} \text {. }
$$

If $i \notin S$, then the output is terminator $\perp$.

(b) If $x$ is a nonleaf node, then for all child nodes $z$ of $x$, recursively call algorithm DecryptNode $(C T$, $S K, z)$ and calculate $F_{z}=$ DecryptNode $(C T, S K, z)$. For example, let us suppose that $S_{x}$ is an arbitrary node set that is composed of exactly $k_{x}$ child nodes. Then, for each node $z$ in $S_{x}$, the condition $F_{z} \neq \perp$ is satisfied. If this condition does not exist, the terminator $\perp$ is the output.

If $i \notin S$, then the output is terminator $\perp$.

(b) If $x$ is a nonleaf node, then for all child nodes $z$ of $x$, recursively call algorithm DecryptNode $(C T, S K, z)$ and calculate $F_{z}=$ DecryptNode $(C T, S K, z)$. For example, let us suppose that $S_{x}$ is an arbitrary node set that is composed of exactly $k_{x}$ child nodes. Then, for each node $z$ in $S_{x}$, the condition $F_{z} \neq \perp$ is satisfied. If this condition does not exist, the terminator $\perp$ is the output.

(b) If $x$ is a nonleaf node, then for all child nodes $z$ of $x$, recursively call algorithm DecryptNode $(C T, S K, z)$ and calculate $F_{z}=$ DecryptNode $(C T, S K, z)$. For example, let us suppose that $S_{x}$ is an arbitrary node set that is composed of exactly $k_{x}$ child nodes. Then, for each node $z$ in $S_{x}$, the condition $F_{z} \neq \perp$ is satisfied. If this condition does not exist, the terminator $\perp$ is the output.

\section{System Performance Verification}

The test environment and various parameters and assumptions of the system are shown in Table 1.

After logging into the system, verify the working performance of the system. The specific verification results are as follows.

5.1. Various Information Function Tests. The test results of various information functions of the system are shown in Table 2.

From the system performance test results shown in Table 2, it can be observed that the overall operation results of the system are basically consistent with the expected results, indicating that the designed system can effectively manage university science and technology projects.

5.2. System Data Reading Completeness. Since the system needs frequent data entry and reading and the entry is entered manually, the completeness of information extraction needs to be verified. The system data speed completeness index is a parameter to measure whether the data reading of the management system is complete. One complete reading is recorded as 1 point, and if it cannot be completed, it will not be scored. The corresponding system data reading completion index is obtained by calculating the conditional ratio between the score and the 
TABle 1: System test environment.

\begin{tabular}{lcc}
\hline Tools/environment & Name & Version \\
\hline Web application server & Apache Tomcat & 7.0 \\
Database & Oracle & $11 \mathrm{gR} 2$ \\
& Junit & 4.11 \\
Test framework & Dbunit & 2.5 .1 \\
& Spring-test & 3.2 .2 release \\
Client side & Easymock & 3.1 \\
Testing tool & Browser & IE, Chrome 41, and Firefox 36.04 \\
& Firebug & 2.0 \\
& Chrome DevTools & 41
\end{tabular}

TABLE 2: Information test forms.

Test purpose: test the correctness of business process flow and function realization in the basic information management module of the project

Project information management module

Input information

(1) Log in to the system and enter

the system home page

(2) Select project information

management connection

(3) Select the right triangle button below the item list

(4) Select the right double triangle button below the item list

(5) Select any data connection in the paging link below the item list

(6) Enter the project information management page

(7) Click the add button

(8) Enter basic project information
Tester

Prediction results

Open the item query list, and the left triangle button below the item list is gray

Paginate the project records on the last page

Paginate the project records on the last page

The project records on the last page are displayed The project records on the last page are displayed in pages, and the right double-triangle button is in pages, and the right double-triangle button is gray

Pagination displays the connection records on the specified page

Open the add project basic information page

Pagination displays the connection records on the specified page

Open the add project basic information page Prompt success after entry
Prompt success after entry

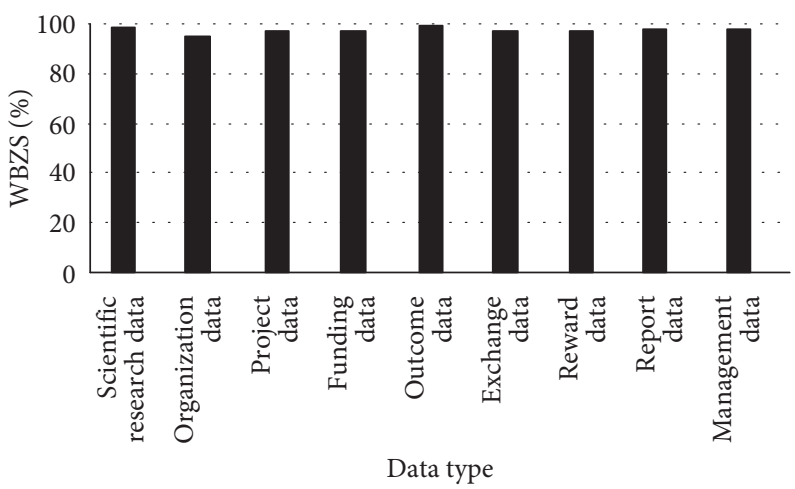

FIGURE 8: Data reading completeness results.

total test times, expressed in WBZS. Wherein, WBZS is greater than $60 \%$, indicating that the system can perform basic reading; WBZS is greater than $80 \%$, which means that the system can read data well; WBZS is greater than $90 \%$, which means that the system can fully complete data reading; WBZS is lower than $50 \%$, which means that the system cannot complete data reading. The data reading completeness results of multiple science and technology projects are shown in Figure 8.

It can be seen from the experimental results shown in Figure 8 that the WBZS of the designed system is always maintained at more than $90 \%$ for different data types, 
indicating that the designed system can read all data types completely, indicating that the management performance of the system is strong.

\section{Conclusions and Future Work}

It is an inevitable trend for universities to realize the informatization of scientific research project management. On the one hand, the introduction of information technology management mode can greatly reduce the workload of scientific research project managers and standardize and simplify the management process, and on the other hand, it can also use advanced database management systems to improve management efficiency and accuracy and improve management levels. The paper covers the importance of a single repository for updating and maintaining a document management system so that stakeholders can access and use the material for their respective purposes on a regular basis. The system is developed in the $\mathrm{B} / \mathrm{S}$ mode, and the J2EE architecture is introduced. With the support of the information system, the scientific, standardized, and informatized practical scientific research project management can be better realized. The next research direction is to add intelligent automatic evaluation functions to the system to enhance the ease of use and operability of the system. In the future, we have plan to design a dynamic report generating module that will be able to generate all types of reports from the front-end without the need for any developer participation. Users have also requested graphical analysis of the data, so we aim to include that as well.

\section{Data Availability}

The data used to support the findings of this study are available from the corresponding author upon request.

\section{Conflicts of Interest}

The authors declare that they have no conflicts of interest regarding the publication of this paper.

\section{Acknowledgments}

This study was supported by 2021 Jiangsu University Philosophy and Social Science Research General Project "Study on the Path to Cultivating the Artisan Spirit of Higher Vocational Talents under the Model of School-Enterprise Cooperation" (2021SJA2124), Moderator: Liu Jinhua; 2021 Jiangsu Provincial Science Coordination Research Project "Jiangsu Agricultural Higher Vocational College Service Research on the Development Path of Local Agriculture" (JSKXKT2021047), Moderator: Liu Jinhua; and 2021 Science and Technology Project of Jiangsu Agriculture and Forestry Vocational College "Study on the Cultivation Strategy of Craftsman Spirit in Higher Vocational School-Enterprise Cooperation" (2021kj58).

\section{References}

[1] L. Chen, "Design and implementation of scientific research management system in higher vocational colleges based on data depth analysis," Digital Technology \& Application, vol. 37, no. 10, pp. 161-162, 2019.

[2] Y. Wu, C. Yue, and J. Jing, "Thoughts on construction of the secondary-tier scientific research management system in colleges and universities," Science Research Management, vol. 40, no. 12, pp. 327-330, 2019.

[3] G. Wang and Y. Wang, "Innovative marketing framework for enterprises using edge-enabled data analysis," Mobile Information Systems, vol. 2021, Article ID 6699420, 8 pages, 2021.

[4] H. Niu, "Innovation of scientific research management and data management system in universities under big data environment," Modern Information Technology, vol. 2, no. 3, pp. 115-117, 2018.

[5] Z. Deng, M. Yan, and X. Xiao, "An early risk warning of crossborder E-commerce using BP neural network," Mobile Information Systems, vol. 2021, Article ID 5518424, 8 pages, 2021.

[6] H. J. Ouyang, M. Ge, and L. L. Wang, "Design and implementation of scientific research management system based on ESMSH framework," Computing Technology and Automation, vol. 39, no. 1, pp. 169-173, 2020.

[7] X. Yu, Y. Chu, F. Jiang, Y. Guo, and D. Gong, "SVMs classification based two-side cross domain collaborative filtering by inferring intrinsic user and item features," KnowledgeBased Systems, vol. 141, pp. 80-91, 2018.

[8] H. Zhongsheng, C. Yuhao, Y. Ming et al., "Bayesian method based on the equipment level electromagnetic pulse effect assessment," Intense Laser and Particle Beam, no. 12, pp. 243-247, 2015.

[9] L. Yu, H. Feng, and L. Xicheng, "Bayesian estimation of the confidence lower limit of electromagnetic pulse vulnerability in polymorphic systems," Modern Applied Physics, vol. 7, no. 3, pp. 1-6, 2016.

[10] X. Du, "Research on engineering project management method based on BIM technology," Scientific Programming, vol. 2021, Article ID 7230585, 10 pages, 2021.

[11] C. Mengcheng, F. Wei, Y. Chao, and X. Li, "Bayes statistics and MCMC method-Matlab program realization of metropolis-Hastings (mh) algorithm," Journal of east China Jiaotong University, vol. 35, no. 1, pp. 1-8, 2018.

[12] X. Yu, J. Yang, and Z. Xie, "Training SVMs on a bound vectors set based on fisher projection," Frontiers of Computer Science, vol. 8, no. 5, pp. 793-806, 2014.

[13] T. Lingiiao, H. Bo, L. Peitao, and Z. Huiqiu, "Bayesian estimation of electromagnetic protection performance of electronic systems," Automation Command \& Computer, no. 1, pp. 5-9, 2011.

[14] M. Yu, T. Quan, Q. Peng, X. Yu, and L. Liu, "A model-based collaborate filtering algorithm based on stacked autoencoder," Neural Computing \& Applications, vol. 6, 2021.

[15] Y. Han, "Exploration of precise management system for scientific research projects under new national S \& T system-taking administrative offices of CAS as example," Bulletin of Chinese Academy of Sciences, vol. 33, no. 6, pp. 622-629, 2018. 